To be submitted to the SMMIB conference

\title{
Formation of Optical Properties of Intermetallic Nanoclusters Formed by Sequential Ion Implantation
}

\author{
R. A. Zuhr \\ Oak Ridge National Laboratory, Oak Ridge, TN \\ R. H. Magruder, III and T. S. Anderson \\ Vanderbilt University \\ Nashville, TN \\ "The submitted manuscript has been
authored by a contractor of the U.S.
Government under contract No. DE-ACOS-
$960 R 22464$. Accordingly, the U.S.
Government retains a nonexclusive.
reproduce the published form of this
contribution, or allow others to do so, for
U.S. Government purposes." \\ Prepared by the \\ Oak Ridge National Laboratory \\ Oak Ridge, Tennessee 37831 \\ managed by \\ LOCKHEED MARTIN ENERGY RESEARCH CORP. \\ for the \\ U.S. DEPARTMENT OF ENERGY \\ under contract DE-AC05-96OR22464
}

September 1997

DISTRIBUTION OF THIS DOCUMENT IS UNLWITES

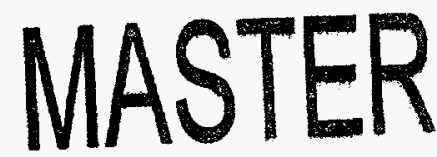




\section{DISCLAIMER}

This report was prepared as an account of work sponsored by an agency of the United States Government. Neither the United States Government nor any agency thereof, nor any of their employees, makes any warranty, express or implied, or assumes any legal liability or responsibility for the accuracy, completeness, or usefulness of any information, apparatus, product, or process disciosed, or represents that its use would not infringe privately owned rights. Reference herein to any speciric commercial product, process, or service by trade name, trademark, manufacturer, or otherwise does not necessarily constitute or imply its endorsement, recommendation, or favoring by the United States Government or any agency thereof. The views and opinions of authors expressed herein do not necessarily state or reflect those of the United States Government or any agency thereof. 


\title{
Formation and Optical Properties of Intermetallic Nanoclusters Formed by Sequential Ion Implantation
}

\author{
R. A. Zuhr \\ Solid State Division, Oak Ridge National Laboratory \\ Oak Ridge, TN 37831 \\ R. H. Magruder, III, and T. S. Anderson \\ Dept. of Applied and Engineering Sciences \\ Vanderbilt University \\ Nashville, TN 37235
}

\begin{abstract}
Recent demonstrations that large third order nonlinear responses can be achieved in metal nanocluster glass composites are of significant interest because of their potential for use in all optical switching networks. These composite materials exhibit picosecond switching and relaxation times, thermal and chemical stability, high laser damage thresholds, and low two photon absorption. Ion implantation has been shown to be a useful fabrication method to form these nanoclusters in silica because of its ability to produce thin films in waveguide configurations containing a high volume fraction $(>1 \%)$ of metal colloids with well defined vertical and horizontal dimensional control. Using sequential ion implantation of more than one element we can modify the composition and microstructure of the composites by forming intermetallic metal colloids. In this work we report on the improved optical response of metallic nanocluster composites formed by sequential implantation of $\mathrm{Cd}$ and $\mathrm{Ag}$ and $\mathrm{Sb}$ and $\mathrm{Ag}$.

Characterization of the samples by transmission electron microscopy (TEM) reveals that approximately spherical metallic colloids are formed for all implanted species during the implantation process. Selected area diffraction patterns indicate that the colloids formed
\end{abstract}


are intermetallic in composition. Linear optical absorption measurements made at room temperature in air from 900 to $200 \mathrm{~nm}$ show significant changes in both the magnitude and wavelength of the surface plasmon resonance. The formation of intermetallic nanoclusters results in changes in both the linear and nonlinear optical properties of the composite material that are not possible with single element colloids alone. The results are explained in terms of effective medium theory. 


\section{INTRODUCTION}

Recent demonstrations that large third order nonlinear responses can be achieved in metal nanocluster glass composites are of significant interest because of their potential for use in all optical switching networks. These composite materials exhibit picosecond switching and relaxation times, thermal and chemical stability, high laser damage threshold and low two photon absorption. Ion implantation has been shown to be a useful fabrication method to form these nanoclusters in silica because of the possibility of forming thin films in waveguide configurations containing a high volume fraction $(>1 \%)$ of metal colloids with well defined vertical and horizontal dimensional control.

Arnold and Borders 1 initially used this technique to form Ag and Au colloids in silicate glasses while others have used this technique to form $\mathrm{Au}, \mathrm{Ag}, \mathrm{Sn}, \mathrm{Cu}, \mathrm{Sb}, \mathrm{Pb}, \mathrm{Bi}$, $\mathrm{Ti}, \mathrm{Fe}, \mathrm{Cr}, \mathrm{Mn}$, and $\mathrm{Ga}[2,3,4,5,6,7,8,-9]$ colloids in different glass substrates. It has been shown that particle size can be controlled by the total dose, current density, and substrate temperature. $[10,11]$ The depth of the implanted particles can be controlled by the implantation energy. The formation and size of these colloias wnen rormed by con implantation are highly dependent upon the composition of the substrate. $[12,13]$ Post implantation processes can subsequently be used to alter the size and size distribution of the colloids. [ $\left.{ }^{14}\right]$

Sequential ion implantation can be used to extend the ion implantation method of forming elemental metal nanocluster-glass composites by allowing the formation of multicomponent nanometer dimension metal particles in glass. $[15,16]$ This technique has been demonstrated to significantly alter the composition of the metal particles and thus the electronic structure of the colloids. As a consequence the formation of multicomponent nanoclusters produces changes in both the linear and nonlinear optical properties of the composite that are not possible with single element colloids. $[17,18]$

The optical properties of these metal colloid-glass composites can be described using effective medium theory for small non interacting particles in a dielectric.[1] The 
linear response for colloids with diameters less than $\lambda / 20$, where $\lambda$ is the wavelength of the incident radiation, is reasonably described by effective medium theory in the electric dipole approximation $[1,19]$ and is given by

$$
\alpha=\frac{18 \pi \mathrm{n}_{\mathrm{d}}^{3}}{\lambda} \cdot \frac{\mathrm{p} \varepsilon_{2}}{\left[\varepsilon_{1}+2 \mathrm{n}_{\mathrm{d}}^{2}\right]^{2}+\varepsilon_{2}^{2}},
$$

where $\alpha$ is the absorption coefficient, $\varepsilon=\varepsilon_{1}+i \varepsilon_{2}$ is the dielectric constant of the metal, $p$ is the volume fraction of the metal particles and $n_{d}$ is the index of refraction of the dielectric host. The absorption is expected to exhibit a peak at the surface plasmon resonance

frequency for which the condition $\varepsilon_{1}+2 n_{d}^{2}=0$ is met. The surface plasmon resonance frequency depends on both the electronic properties of the metal colloids and the index of refraction of the host dielectric, $n_{d}$.

As the linear and nonlinear optical properties are dependent on the dielectric function of the nanoclusters, the ability to alter dielectric properties by sequential ion implantation offers a unique way to significantly improve and tailor the optical properties of these composite materials for use in optical devices. Here we review the modification of the optical response of nanometer dimension metal colloids formed by sequential implantation of $\mathrm{Cd} / \mathrm{Ag}$ and $\mathrm{Sb} / \mathrm{Ag}$. Single element nanoclusters were also studied for comparison.

\section{EXPERIMENT}

All sample were made by implantation into Corning 7940 high purity silica substrates. The implantation energies for the $\mathrm{Sb} / \mathrm{Ag}$ and $\mathrm{Cd} / \mathrm{Ag}$ systems were chosen from TRIM 89 calculations to determine energies that would overlay the depths of implantation for each metal.

The first set of samples was formed using $320 \mathrm{keV} \mathrm{Sb}$ at a nominal dose of $3 \times 10^{16}$ ions $/ \mathrm{cm}^{2}$ and $305 \mathrm{keV} \mathrm{Ag}$ at a nominal dose of $9 \times 10^{16}$ ions $/ \mathrm{cm}^{2}$. Implantation of $\mathrm{Sb}$ followed by $\mathrm{Ag}$ at a dose ratio of 3:9 Sb:Ag formed a nanometer dimension colloid-glass 
composite. For comparison single element samples of $\mathrm{Ag}$ and $\mathrm{Sb}$ were implanted at the same doses.

The second series of samples was implanted using $320 \mathrm{keV} \mathrm{Cd}$ followed by 305 $\mathrm{keV} \mathrm{Ag}$, each to the same dose of $6 \times 10^{16}$ ions $/ \mathrm{cm}^{2}$. The dose ratio for these composites was 6:6 Cd:Ag for a total dose of $12 \times 10^{16}$ ions $/ \mathrm{cm}^{2}$. Again single element implantations of $\mathrm{Ag}$ and $\mathrm{Cd}$ were made for comparison.

Rutherford backscattering measurements (RBS) with $2.3 \mathrm{MeV} \mathrm{He}{ }^{++}$ions were used to measure the depth profiles of the implanted species. The size distributions and geometrical structure of the nanoclusters in these composites were determined by transmission electron microscopy (TEM). Sample preparation has been described previously.[17] The samples were examined in a $200-\mathrm{keV}$ scanning transmission electron microscope using bright field (BF) and dark field (DF) imaging, and selected-area electron diffraction (SAD).

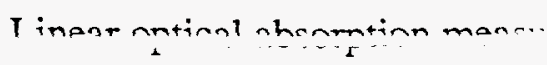

900 to $200 \mathrm{~nm}$ using a dual beam spectrometer (Cary 5). The absorption spectra were measured at three different positions on each sample using an unimplanted sample in the reference beam. The scatter in these measurements at the tifferent positions was less than $1 \%$.

\section{RESULTS}

The $\mathrm{Sb}$ and $\mathrm{Ag}$ were implanted at a dose ratio of $3 \mathrm{Sb}$ to $9 \mathrm{Ag}$ because $\mathrm{Ag}_{3} \mathrm{Sb}$ is a stable compound in the Ag-Sb system. Analysis of the micrograph of the single element $9 \times 10^{16} \mathrm{Ag}$ sample in Figure 1a shows that the particles are spherical in shape with bidispersed particle sizes. Analysis of selected area electron diffraction rings indicate that the particles present are FCC silver. A micrograph of the single element $3 \times 10^{16} \mathrm{Sb}$ implanted sample in Figure $1 \mathrm{~b}$ reveals approximately spherical colloids with much smaller particle sizes than the Ag sample. Electron diffraction patterns of the Sb implanted sample 
are diffuse rings characteristic of noncrystalline $\mathrm{Sb}$. Mean particle sizes for the single element $\mathrm{Ag}$ and $\mathrm{Sb}$ samples are $24.20+/-1.8 \mathrm{~nm}$ and $6.03+/-1.8 \mathrm{~nm}$ respectively. Figure 2 shows a bright field image of the implanted layer for the 3:9 Sb:Ag sample. The microstructure of the 3:9 $\mathrm{Sb}: \mathrm{Ag}$ sample displays approximately spherical colloids with distributed particle sizes and a mean particle size of $9.65 \mathrm{~nm}+/-1.8 \mathrm{~nm}$. Selected area diffraction patterns reveal that colloids in these samples are crystalline. The $d$-spacings are consistent with elemental $\mathrm{Ag}$ and an $\mathrm{Ag}_{3} \mathrm{Sb}$ phase.

Figure 3 shows the optical spectra for the $\mathrm{Ag}, \mathrm{Sb}$, and $\mathrm{Sb} / \mathrm{Ag}$ samples. The absorption spectrum for the $9 \times 10^{16} \mathrm{Ag}$ sample is dominated by an absorption peak at $\sim 400$ $\mathrm{nm}$ and a peak centered at $\sim 610 \mathrm{~nm}$. The spectrum for the $\mathrm{Sb}$ sample displays no resonance peaks, simply increasing absorption with decreasing wavelength. The absorption spectrum for the 3:9 Sb:Ag sample displays two overlapping absorption peaks centered at $\sim 416 \mathrm{~nm}$ and $\sim 470 \mathrm{~nm}$. The optical spectrum of the sequentially implanted sample does not match the spectra observed for the single element samples. However, the peak at $\sim 416 \mathrm{~nm}$ in the 3:9 Sb:Ag sample is close to the $400 \mathrm{~nm}$ absorption peak seen for the single element $\mathrm{Ag}$ sample.

The ratio of $\mathrm{Cd} / \mathrm{Ag}$ of $6 / 6$ was chosen because there are two stable compounds of $\mathrm{Ag}$ and $\mathrm{Cd}, \mathrm{AgCd}$ and $\mathrm{Ag}_{5} \mathrm{Cd}_{8}$ that are near this ratio. Figure 4 shows the $\mathrm{RBS}$ for the $\mathrm{Ag}, \mathrm{Cd}$, and $\mathrm{Cd} / \mathrm{Ag}$. For the single element implantations the distributions as a function of depth from the surface are gaussian in shape with peaks at $\sim 0.14$ microns. The $\mathrm{Ag}$ implanted sample has a narrower distribution and a sharper peak than does the $\mathrm{Cd}$ sample. The full width half maximum (FWHM) is $\sim 0.1$ microns for the Cd implanted sample and $\sim$ 0.09 microns for the Ag implanted sample. The RBS curve for the sequentially implanted $\mathrm{Cd} / \mathrm{Ag}$ sample is a slightly skewed gaussian with a peak at $\sim 0.15$ microns and a FWHM that is the same as the Cd implanted sample. The dose as measured by RBS for the single element implantations is $4.7 \times 10^{16}+/-5 \%$ ions $/ \mathrm{cm}^{2}$ and $5.0 \times 10^{16}+/-5 \%$ ions $/ \mathrm{cm}^{2}$ for the $\mathrm{Ag}$ and $\mathrm{Cd}$ samples respectively. The total dose as measured by RBS for the sequentially 
implanted $\mathrm{Cd} / \mathrm{Ag}$ sample is $1.0 \times 10^{17}+/-5 \%$ ions $/ \mathrm{cm}^{2}$, which agrees within error with the sum of the single element implantations.

Transmission electron micrographs of the single element Ag and $\mathrm{Cd}$ samples in Figure 5 a and b respectively show that spherical particles are formed during the implantation process. The microstructure of the Ag implanted sample displays approximately spherical colloids with a broader particle size distribution than the single element Cd implanted sample. Mean particle size in the Ag sample is $13.5+/-1.8 \mathrm{~nm}$ with a significant number of particles larger than $25 \mathrm{~nm}$. Electron diffraction analysis indicates that the particles present are FCC silver. The microstructure of the Cd sample displays approximately spherical colloids with no particles $>15 \mathrm{~nm}$ in diameter and a mean particle size of $7.2+/-1.8 \mathrm{~nm}$. Electron diffraction reveals that the colloids are polycrystalline $\mathrm{Cd}$.

The transmission electron micrograph of the sequentially implanted $\mathrm{Cd} / \mathrm{Ag}$ sample in Figure 6 displays a high density of approximately spherical colloids with no particles > $25 \mathrm{~nm}$ in diameter and a mean particle size of $8.6+/-1.8 \mathrm{~nm}$. Selected area diffraction patterns show that the colloids are polycrystalline and the $d$-spacings are most consistent with a phase of $\mathrm{Ag}-\mathrm{Cd}, \mathrm{Ag}_{5} \mathrm{Cd}_{8}$, and elemental $\mathrm{Ag}$. Lattice spacings measured directly from the high resolution electron micrograph of Figure 7 are consistent with $\mathrm{Ag}_{5} \mathrm{Cd}_{8}$.

Figure 8 shows the optical spectra for the $\mathrm{Ag}, \mathrm{Cd}$, and $\mathrm{Cd} / \mathrm{Ag}$ implanted samples. The absorption spectrum for the Ag sample is dominated by an absorption peak at $\sim 426 \mathrm{~nm}$ and a shoulder at $\sim 514 \mathrm{~nm}$. The spectrum for the $\mathrm{Cd}$ sample has a resonance peak centered at $\sim 271 \mathrm{~nm}$ with decreasing absorption with decreasing energy. The absorption spectrum for the $\mathrm{Cd} / \mathrm{Ag}$ sample displays two overlapping absorption peaks centered at $\sim 327$ $\mathrm{nm}$ and $\sim 403 \mathrm{~nm}$. The optical spectrum of the Cd/Ag sequentially implanted sample does not match the spectra observed for the single element implanted samples. However, the peak at $\sim 403 \mathrm{~nm}$ in the Cd/Ag sample is close to the $426 \mathrm{~nm}$ absorption peak seen in the optical spectrum of the single element Ag implanted sample. 


\section{DISCUSSION}

During the implantation process both the implanted ions and the silicon matrix compete for the oxygen in the substrate. However, the free energy of formation of $\mathrm{SiO}_{2}$ is lower than the free energy of formation of the oxides of $\mathrm{Ag}, \mathrm{Cd}$, and $\mathrm{Sb} .{ }^{20} \mathrm{Under}$ equilibrium conditions $\mathrm{SiO}_{2}$ is stable with respect to the implanted ions, thus $\mathrm{Ag}, \mathrm{Sb}$ and $\mathrm{Cd}$ remain in metallic form. ${ }^{21}$ Ion implantation generates high local temperatures which provide mobility required for initial colloid formation and serve as a driving force in the diffusional growth process of the colloids. 22

Comparison of the single element implanted samples aids in tunderstanding the microstructures seen in the sequentially implanted samples. Comparison of the $9 \times 10^{16} \mathrm{Ag}$ and $3 \times 10^{16} \mathrm{Sb}$ samples reveals that though the Ag sample contains dramatically larger particles than does the Sb sample, the implanted layer of the Sb sample displays a higher density of particles.

dose ratio of the sequentially implanted sample. Initial implantation of Sb produces a high particle density resulting in two important effects; first, more available nucleation sites, and second, a greater area of reaction interface which $w_{i}$. produce overlapping denuded zones. Because of these two factors the reaction is expected to proceed at a significantly enhanced rate. Based on these effects, the resulting microstructure seen in Figure 3 is similar to the single element Sb implanted samole. However, due to the slower diffusion of $\mathrm{Sb}$ there is a build-up of $\mathrm{Ag}$ ions in the mars esulting in the formation of some $\mathrm{Ag}$ colloids. While most of the d-spacings obtamet trom this sample are consistent with an $\mathrm{Ag}_{3} \mathrm{Sb}$ phase, there are some which correspont the metallic $\mathrm{Ag}$, suggesting that the reaction, though not at equilibrium, has create sufficient amount of $\mathrm{Ag}_{3} \mathrm{Sb}$ to produce diffraction patterns.

The RBS curve for the single element implanted samples in Figure 4 shows that there is a narrower distribution of ions in the Ag implanted sample than in the Cd implanted 
sample. From the RBS spectra it is clear that the two ion species have significaint overlap in the sequentially implanted samples. Comparison of the $\mathrm{Ag}$ and $\mathrm{Cd}$ samples reveals that although the Ag sample contains larger particles, the Cd sample displays a greater number of particles.

For overlapping ion distributions, implanting one ion into a substrate which has been previously implanted with a different ion is similar to slowly adding a second metal to the first metal. Implanting $\mathrm{Cd}$ first followed by the implantation of $\mathrm{Ag}$ is similar to slowly adding $\mathrm{Ag}$ to $\mathrm{Cd}$ metal, with the heat generated by the implantation process serving as a driving force for the mixing of the ions. From the binary alloy phase diagram of $\mathrm{Ag}$ and $\mathrm{Cd}$ there are two equilibrium compounds with compositions $\mathrm{AgCd}$ and $\mathrm{Ag}_{5} \mathrm{Cd}_{8}$. Following the phase diagram of $\mathrm{Ag}-\mathrm{Cd}$ the first equilibrium compound reached in this direction is $\mathrm{Ag}_{5} \mathrm{Cd}_{8}$. From electron diffraction measurements the sequential implantation of $\mathrm{Cd}$ followed by $\mathrm{Ag}$ results in the formation of a $\mathrm{Ag}$-Cd intermetallic phase, $\mathrm{Ag}_{5} \mathrm{Cd}_{8}$, and elemental Ag. Likewise, lattice spacings measured from HRTEM shown in Figure 7 are consistent with $\mathrm{Ag}_{5} \mathrm{Cd}_{8}$.

There are two mechanisms of absorption in metal colloid-glass composites. The first mechanism is due to defects created during implantation, and the second mechanism is the interaction of the metal colloids with the incident radiation. In the first case absorption is due to defects generated during implantation, such as the $B_{2}, E^{\prime}$, peroxy, and homobond centers. These defects have broad absorption bands in silica at 5, 5.8, 7.5, and $7.6 \mathrm{eV}$ respectively. 23,24 However, the absorption due to these defects is expected to be dominated by the absorption due to the metal colloids at the metal concentrations used in these samples.

From equation (1) the surface plasmon resonance will occur where the condition $\varepsilon_{1}+2 n_{d}^{2}=0$ is met. Also from equation (1) the height and width of the resonance peak is determined by the value of $\varepsilon_{2}$, which in turn depends on the electronic structure of the metal nanoclusters and is size dependent. For metals which do not exhibit free electron behavior, 
yet still display resonance peaks in the absorption spectra, some interband excitation is also included with the surface plasmon resonances. ${ }^{25}$ It is therefore reasonable to expect features of the absorption spectra, as well as the magnitudes, to vary with the electronic structure of the implanted elements. Off the surface plasmon resonance frequency Mie theory predicts that absorption should increase with decreasing wavelength.

For the $\mathrm{Sb} / \mathrm{Ag}$ system the single element $\mathrm{Ag}$ implanted sample has absorption peaks at $\sim 400 \mathrm{~nm}$ and $\sim 610 \mathrm{~nm}$. The peak at $400 \mathrm{~nm}$ is attributed to the surface plasmon resonance of the Ag particles $<20 \mathrm{~nm}$. The peak at $\sim 610 \mathrm{~nm}$ is attributed to the surface plasmon resonance of the largest $\mathrm{Ag}$ particles producing multipole resonances. ${ }^{26}$ For the single element $\mathrm{Sb}$ implanted sample the conditions for resonance are not met. Increasing absorption with decreasing wavelength is exhibited in both single element implanted samples as predicted by Mie theory. The values observed for the surface plasmon resonance for the single element implanted samples are consistent with previously reported values ${ }^{23}$ The absorption spectrum for the sequentially implanted 3:9 Sb:Ag sample displays two overlapping peaks positioned at $\sim 416 \mathrm{~nm}$ and $\sim 470 \mathrm{~nm}$ as seen in Figure 3 . A simple curve fit, made by adding together background absorption with a $1 / \lambda$ dependence expected for nanometer dimension colloids and two Ga. n peaks centered at $403 \mathrm{~nm}$ and $485 \mathrm{~nm}$ reproduces the absorption spectrum of the $\mathrm{s}, \mathrm{Sb}: \mathrm{Ag}$ sample. This curve fit, shown in Figure 9, demonstrates that the absorption spectrum for the sequentially implanted 3:9 Sb:Ag sample contains two resonance peaks centered at $403 \mathrm{~nm}$ and $485 \mathrm{~nm}$. No higher order resonance peaks are expectes :.: his sample due to the absence of large particles. We attribute the peak at $\sim \$ 03 \mathrm{~nm}: 2=$ surface plasmon resonance of the $\mathrm{Ag}$ particles, and the peak at $\sim 485 \mathrm{~nm}$ to the compound $\mathrm{Ag}_{3} \mathrm{Sb}$. The presence of two resnnce peaks suggests that the peritectic reaction expected to take place for this compostion. though not reaching equilibrium (due to the presence of the Ag resonance peak), has produced enough of the metallic compound to display a resonance peak dependent upon $\varepsilon_{1}$ of $\mathrm{A}_{3} \mathrm{Sb}$. 
For the $\mathrm{Cd} / \mathrm{Ag}$ system the optical spectrum of the single element $\mathrm{Ag}$ implanted glass exhibits characteristic absorption due to the surface plasmon resonance of nanometer dimension $\mathrm{Ag}$ colloids as discussed above.[23] We attribute the peak at $426 \mathrm{~nm}$ in the $\mathrm{Ag}$ sample to $\mathrm{Ag}$ colloids $<25 \mathrm{~nm}$ in diameter. The shift in the peak is due to the presence of the larger particles. The absorption shoulder at $515 \mathrm{~nm}$ we attribute to an additional higher order absorption term in Mie theory, i.e., the quadrupole term which also results from the presence of the larger particles.[20] The optical spectrum for the single element $\mathrm{Cd}$ implanted sample exhibits a peak at $271 \mathrm{~nm}$. $\mathrm{Cd}^{+}$exhibits an intense optical band in the UV region. However, all of the Cd is present in the sample as polycrystalline colloids. Therefore we attribute this peak to the surface plasmon resonance of the small metallic nanoclusters of Cd observed in the TEM micrographs. All nanoclusters were less than 20 $\mathrm{nm}$ in diameter and we expect only a dipole absorption term. The optical spectrum of the $\mathrm{Cd} / \mathrm{Ag}$ sample seen in Figure 8 suggests the presence of two peaks. A curve fit with a absorption, gives a reasonable fit to the data as seen in Figure 10. Therefore we attribute the absorption in the $\mathrm{Cd} / \mathrm{Ag}$ implanted sample to the superposition of a peak centered at $\sim 310 \mathrm{~nm}$ due to the formation of $\mathrm{Ag}_{5} \mathrm{Cd}_{8}$ and a peak at $\sim 413 \mathrm{~nm}$ due to elemental $\mathrm{Ag}$. These assignments are consistent with electron diffraction observations. The values observed for the surface plasmon resonance for the single element implanted samples are consistent with previously reported values.[22]

\section{CONCLUSION}

Sequential ion implantation can be used to extend the ion implantation method of forming metal nanocluster glass composites by permitting the fabrication of intermetallic nanoclusters. In general the formation of intermetallics depends on the existence of stable compounds of the two metal species, as in the case of the $\mathrm{Ag} / \mathrm{Sb}$ and $\mathrm{Ag} / \mathrm{Cd}$ systems. To a first approximation phase diagrams can be used to predict the product obtained. Changes 
in the optical properties of the composite materials are caused by changes in the dielectric function of the metal nanoclusters as the intermetallic phases are formed. These nanoclusters have distinctly different optical properties than either of their single element counterparts due to the unique dielectric function that is produced in the intermetallic nanoclusters. The observed changes in the optical properties of these $\mathrm{Ag} / \mathrm{Cd}$ and $\mathrm{Ag} / \mathrm{Sb}$ composite materials are consistent with the predictions of effective medium theory for changes in the dielectric function of the intermetallic clusters.

\section{ACKNOWLEDGMENT}

The authors acknowledge the support of Oak Ridge National Laboratory, managed by Lockheed Martin Energy Research Corp. for the U.S. Department of Energy under contract number DE-AC05-96OR22464 and the support of the Army Research Office under grant DAAH04-93-G-0123 and DAAH04-94-G-0147.

1. G.W. Arnold and J.A. Borders, J. Appl. Phys. 489 (1977) 1488.

2. G. Whichard, H. Hosono, R.A. Weeks, R.A. Zuhr and R.H. Magruder,III, J. Appl. Physics, 67 (1990) 7526.

3. Y. Takeda, T. Hioki, T. Mohohiro, S. Nod d T. Kurauchi, Nuc. Inst. Meth. B 91 (1994) 515.

4. Z. Pan, S.H. Morgan, D.O. Henderson. S. Park, R.A. Weeks, R.H. Magruder,III and R. A. Zuhr, Mat. Res. Soc. Symp. Proc. vol. 316 (1994) 469.

5. R.H. Magruder,III, D. O. Henderson. S. H. Morgan and R. A. Zuhr, J. Non Cryst. Solids, 152 (1993) 258.

6. R.H. Magruder,III, R.A. Weeks, R.A. Zuhr and G. Whichard, J. NonCryst. Solids, 129 (1991) 46. 
7. D.E. Hole, P.D. Townsend, J.D. Barton, L. C. Nistor and J. Van Landuyt, J. Non-Cryst. Solids, 180 (1995) 266.

8. L.C. Nistor, J. Van Landuyt, J.D. Barton, D.E. Hole, N.D. Skelland and P.D. Townsend, J. Non. Cryst. Solids, 162 (1993) 217 .

9. J. Stark, R.A. Weeks, D.L. Kinser and R.A. Zuhr, J. Non-Cryst. Solids, $95 \& 96$ (1987) 685 .

10. C.W. White, D.S. Zhou, J.D. Budai, R.A. Zuhr, R.H. Magruder,III and D.H. Osborne, Mat. Res. Soc. Proc. vol. 316 (1994) 499 .

11. R.H. Magruder,III, R.F. Haglund, L. Yang, J. E. Wittig and R.A. Zuhr, J. of Applied Physics, 76 (1994) 708.

12. A recent review is given by R. A. Weeks, Materials Science and Technology, vol. 9, J. Zarzychi, ed., VCH, Weinheim (1991).

13. P. Mazzoldi, G.W. Arnold G. Bertoncello and F. Gonella, Nuc. Instr. Meth. B 91 (1994) 478.

14. R.A. Wood, P.D. Townsend, N.D. Skelland, D.E. Hole, J. Barton and C.N. Afonso, J. Appl. Phys., 74 (1993) 5754.

15. R. H. Magruder,III, J.E. Wittig and R.A. Zuhr, J. Non Cryst. Solids, 163 (1993) 162.

16 R.A. Zuhr, R.H. Magruder,III and J.E. Wittig, Mat. Res. Soc. Sym. Proc., 316 (1994) 457.

17. R.H. Magruder, III, T.S. Anderson, R.A. Zuhr and D. Thomas.

Nuclear Instruments and Methods B 108 (1996) 305.

18. T.S. Anderson, R.H. Magruder, III, D.L. Kinser, R.A. Zuhr and D.

Thomas, accepted Nuclear Instruments and Methods B.

19. C.F. Bohren and D.R. Huffman, Absorption and Scattering of Light by Small Particles, John Wiley and Sons, New York (1983) . 
20. Thomas B. Reed, Free Energy of Formation of Binary Compounds, 7 - 9 (MIT Press, Cambridge Mass. 1971)

21. H. Hosono, Jpn. J. Appl. Phys. 32, 3892 - 3894 (1993).

22. P.D. Townsend, Rep. Prog. Phys. 50, 501 - 558 (1987).

23. G.W. Arnold and P. Mazzoldi, Ion Beam Modification of Glasses, P. Mazzoldi and G.W. Arnold, eds. 195 - 222 (Elsevier, Amsterdam 1987)

24. R.A. Weeks, Materials Science and Technology vol 9, J. Zarzycki, ed. 331 - 373 (VCH, Weinheim 1991)

25. J. Alan Creighton and D.G. Eadon, Chem. Soc. Faraday Trans. 87, 3881 - 3891 (1991).

26. T.S. Anderson, R.H. Magruder III, R.A. Zuhr, and J.E. Wittig, J. Electronic Mat. 25, $27-33$ (1996). 


\section{FIGURE CAPTIONS}

Figure 1 a) Electron transmission micrograph for 9Ag sample, b) electron transmission micrograph for 3Sb sample.

Figure 2 Electron transmission micrograph for 3Sb/9Ag sample.

Figure 3 Optical density as a function of wavelength for $9 \mathrm{Ag}, 3 \mathrm{Sb}$ and $3 \mathrm{Sb} / 9 \mathrm{Ag}$ samples.

Figure 4 RBS ion distributions as a function of depth for the $6 \mathrm{Ag}, 6 \mathrm{Cd}$ and $6 \mathrm{Cd} / 6 \mathrm{Ag}$ samples.

Figure 5 a) Electron transmission micrograph for $6 \mathrm{Ag}$ sample, b) electron transmission micrograph for $6 \mathrm{Cd}$ sample.

Figure 6 Electron transmission micrographs for $6 \mathrm{Cd} / 6 \mathrm{Ag}$ sample.

Figure 7 High resolution electron transmission micrograph for $6 \mathrm{Cd} / 6 \mathrm{Ag}$ sample.

Figure 8 Optical density as a function of wavelength for $6 \mathrm{Cd}, 6 \mathrm{Ag}$ and $6 \mathrm{Cd} / 6 \mathrm{Ag}$ samples.

Figure 9 Optical density as a function of wavelength for $3 \mathrm{Sb} / 9 \mathrm{Ag}$ and fit with the superposition of peaks at 403 and $485 \mathrm{~nm}$.

Figure 10 Optical density as a function of wavelength for $6 \mathrm{Cd} / 6 \mathrm{Ag}$ and fit with the superposition of peaks at 310 and $412 \mathrm{~nm}$. 

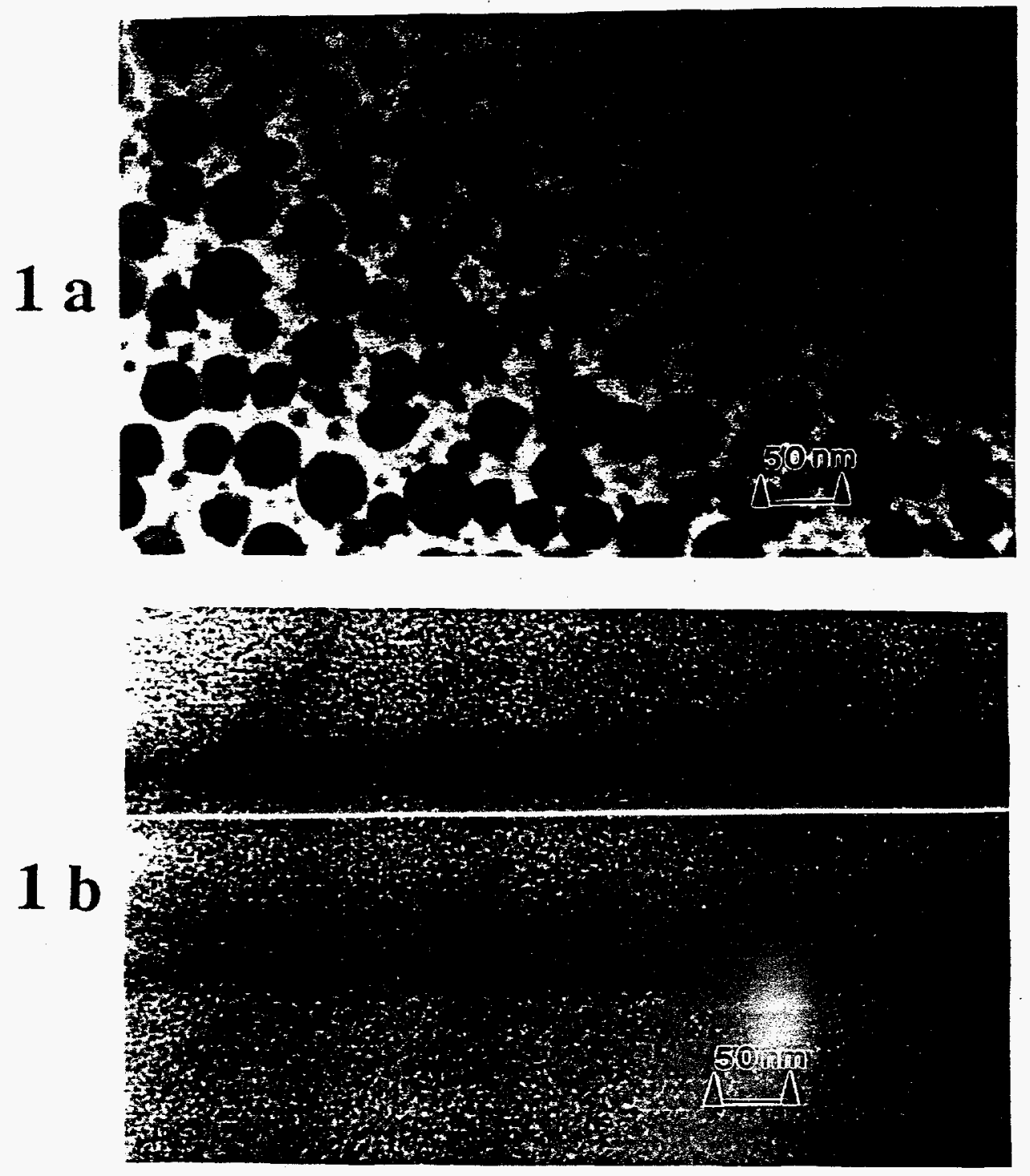


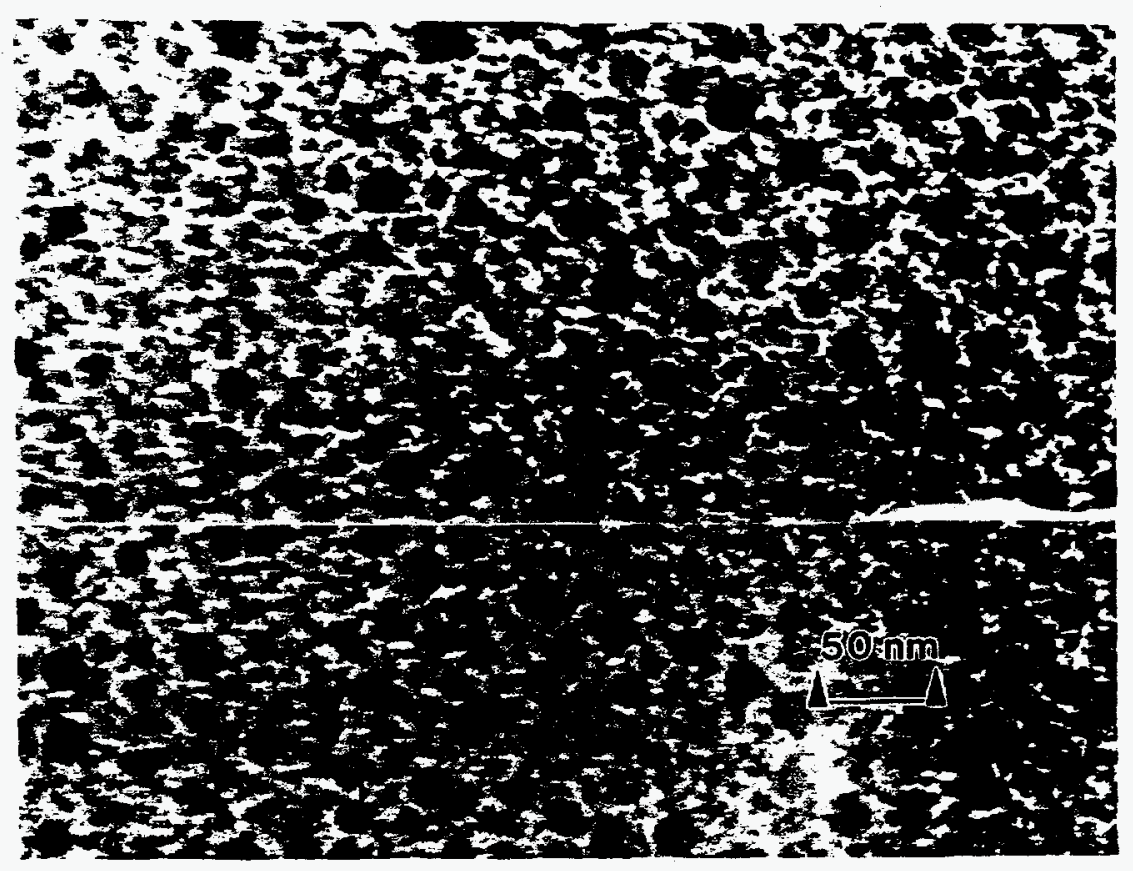




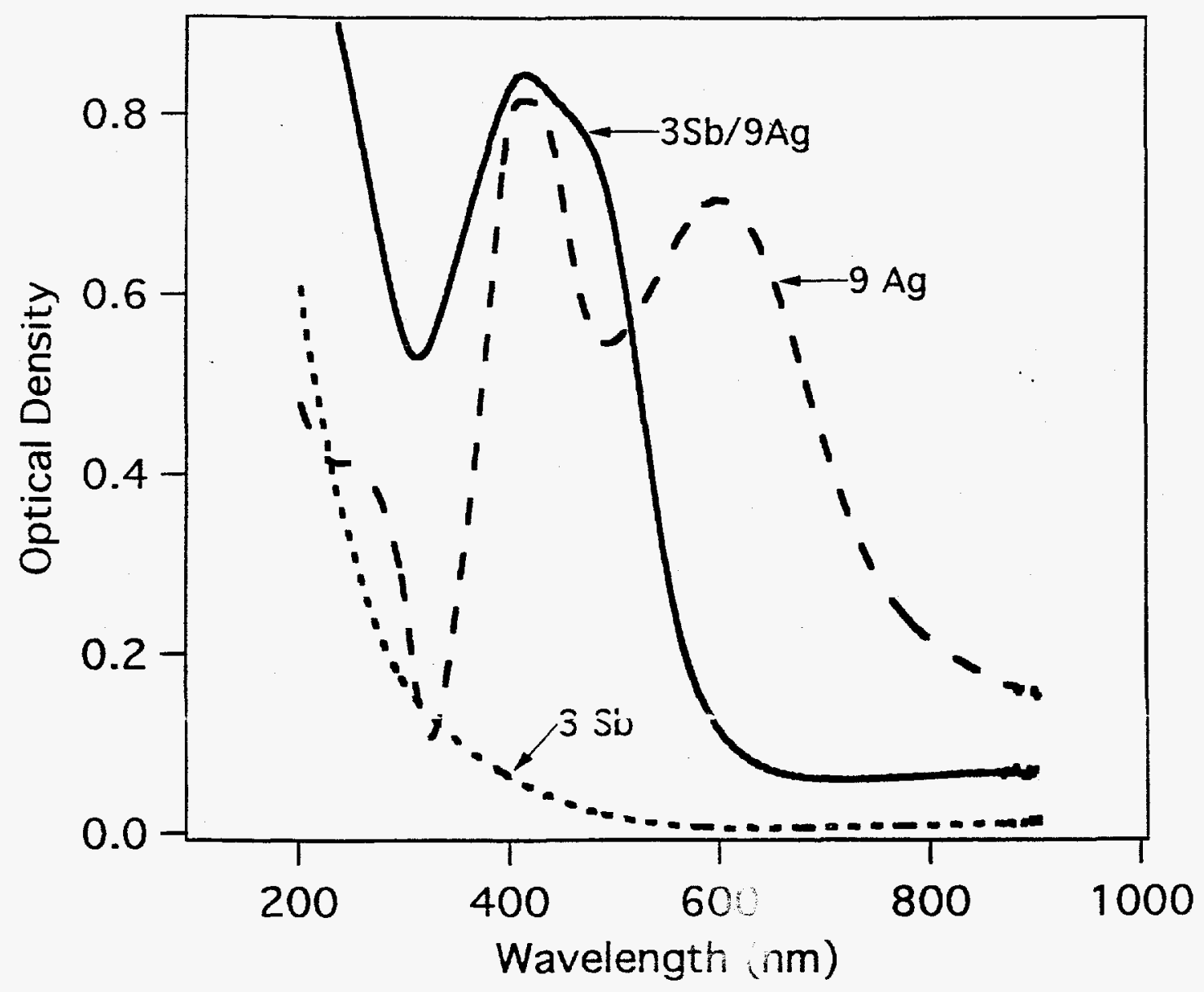

… $\quad 2$

$2 \ldots \therefore \quad-$ 


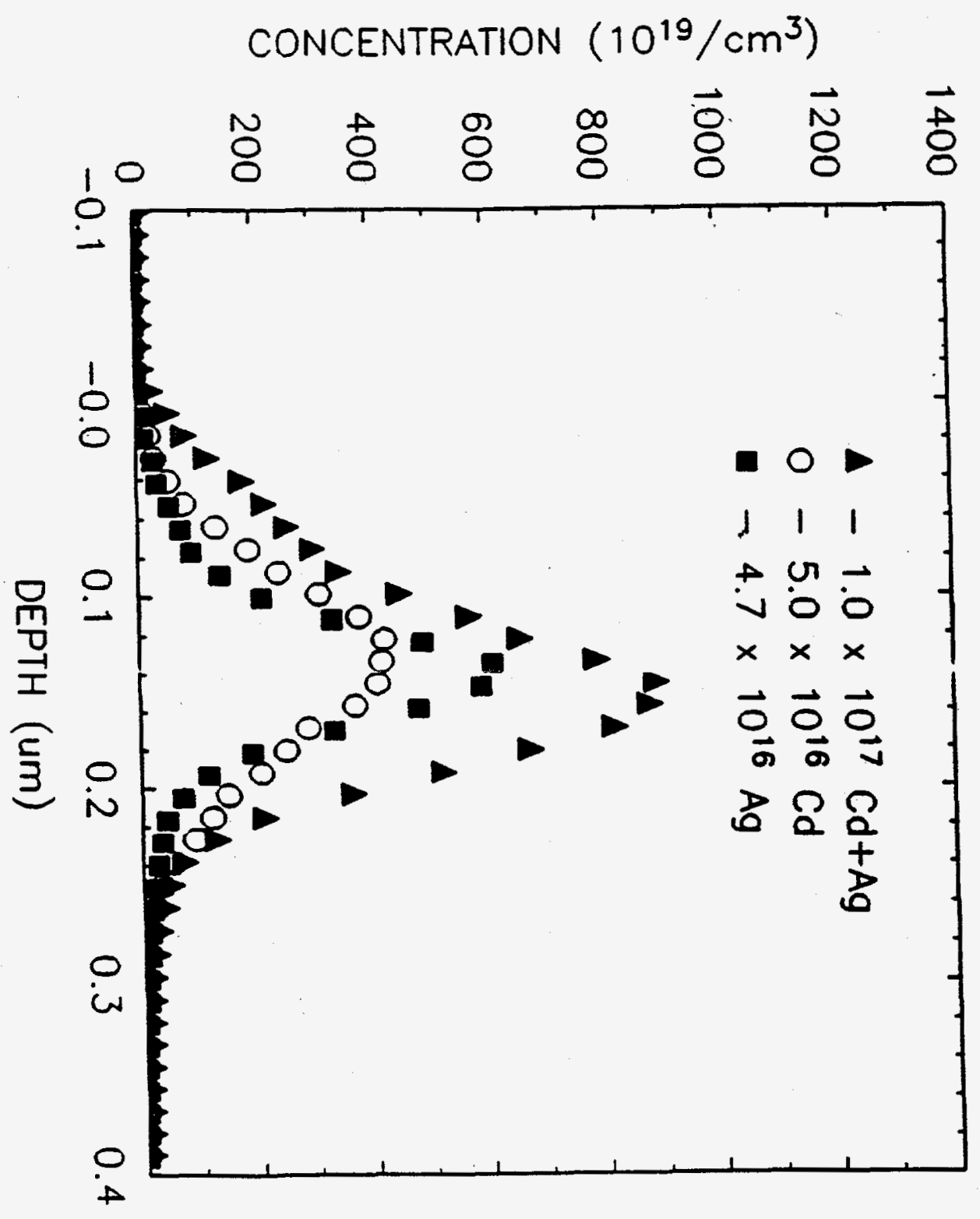

Figure4
zul- 

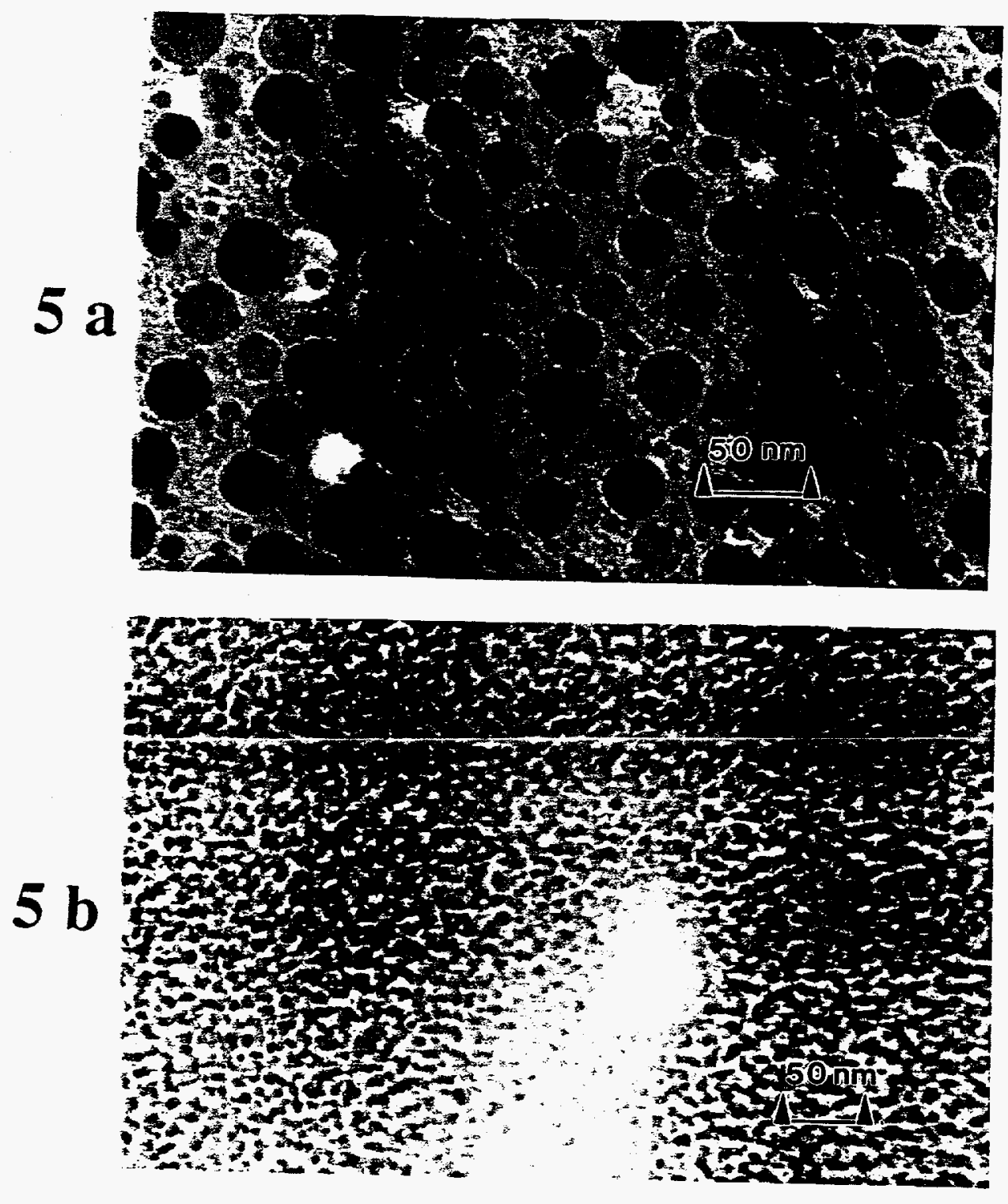


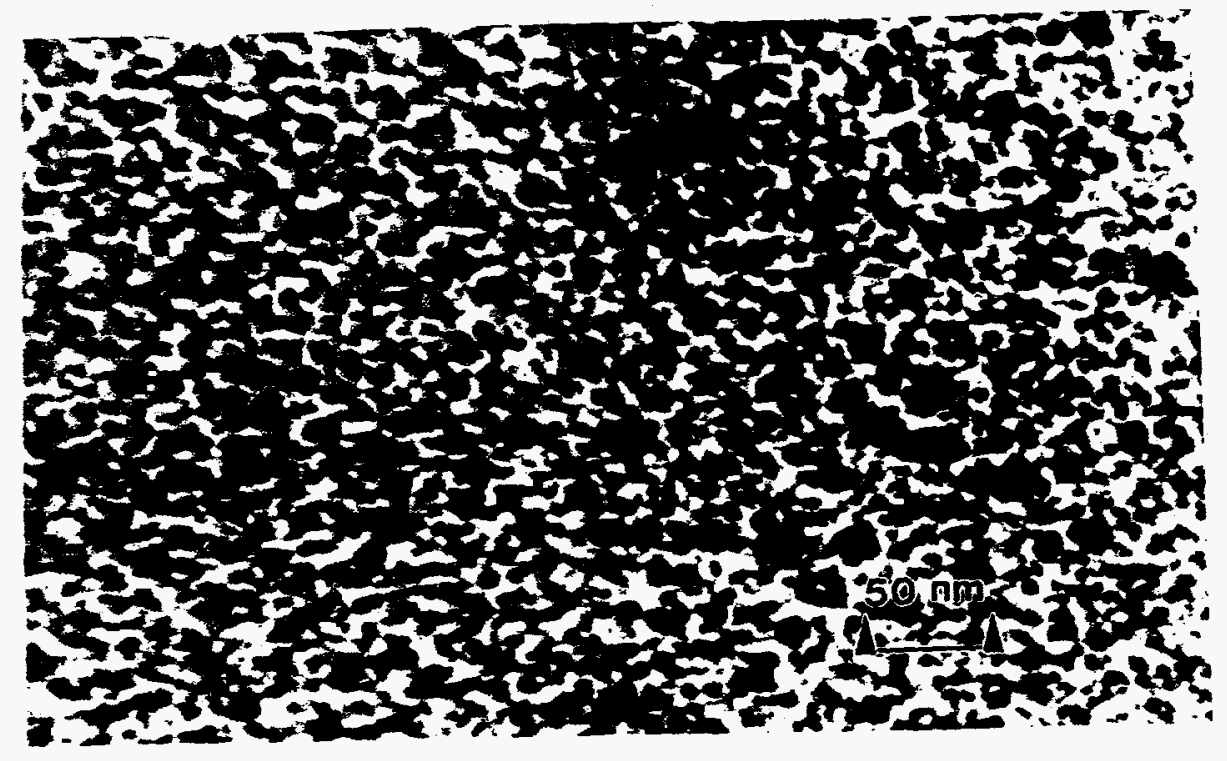




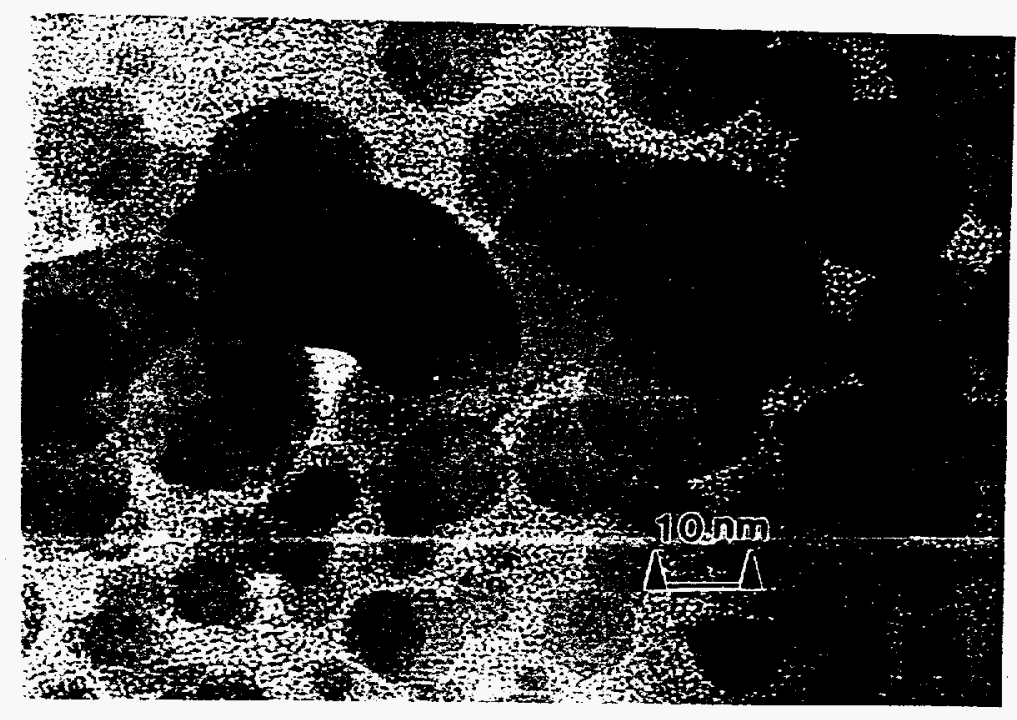




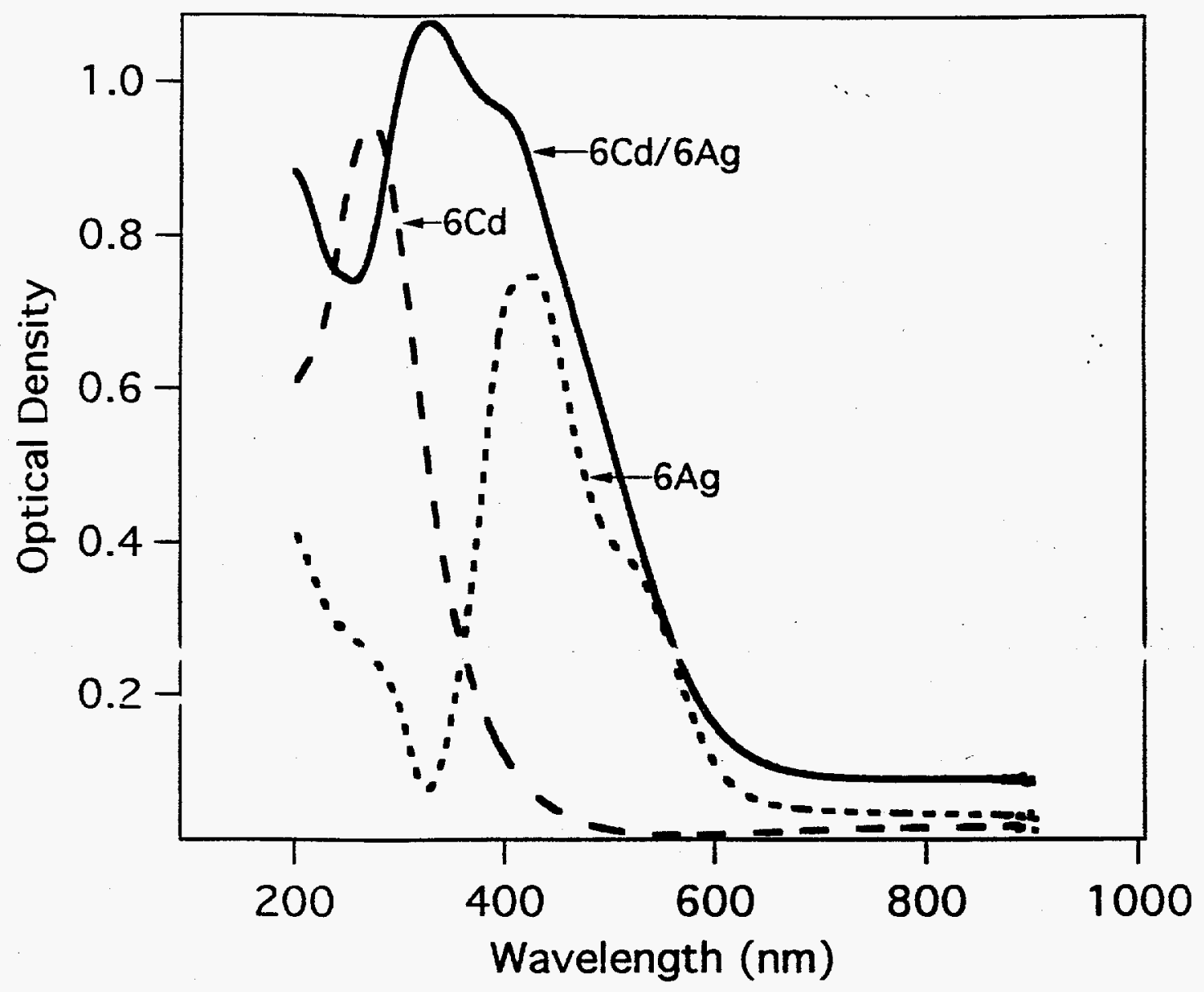

$5 . .188$ 


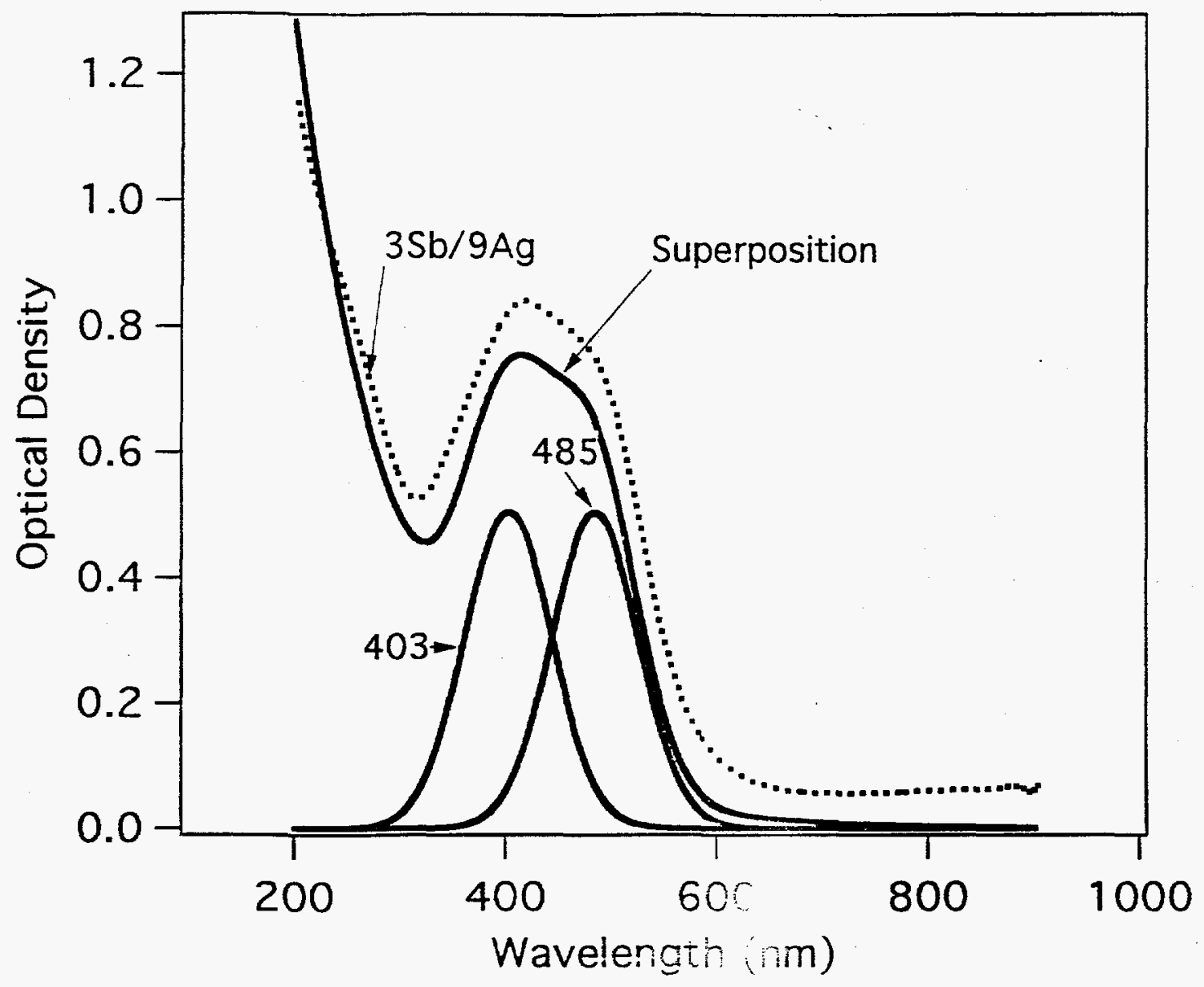

Fi: 
$-\quad \div \quad \leq$

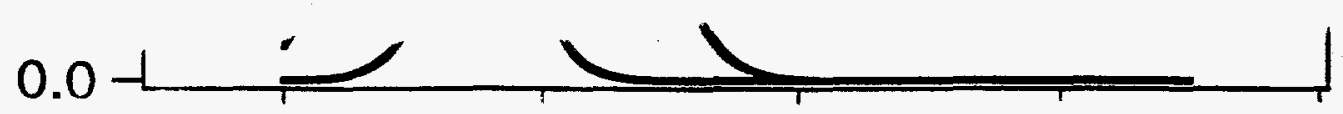

$\cdots+2$ 